\title{
Decomposition of Point-Symmetry Using Ordinal Quasi Point-Symmetry for Ordinal Multi-Way Tables
}

\author{
Yusuke Saigusa, Kouji Tahata, Sadao Tomizawa \\ Department of Information Sciences, Tokyo University of Science, Chiba, Japan \\ Email: saigusaysk@gmail.com,kouji_tahata@is.noda.tus.ac.jp, tomizawa@is.noda.tus.ac.jp
}

Received 12 April 2016; accepted 5 June 2016; published 8 June 2016

Copyright (C) 2016 by authors and Scientific Research Publishing Inc.

This work is licensed under the Creative Commons Attribution International License (CC BY).

http://creativecommons.org/licenses/by/4.0/

(c) ()

\begin{abstract}
For multi-way tables with ordered categories, the present paper gives a decomposition of the point-symmetry model into the ordinal quasi point-symmetry and equality of point-symmetric marginal moments. The ordinal quasi point-symmetry model indicates asymmetry for cell probabilities with respect to the center point in the table.
\end{abstract}

Keywords

Decomposition, Multi-Way Table, Ordinal Quasi Point-Symmetry, Point-Symmetry

\section{Introduction}

Consider an $R_{1} \times R_{2} \times \cdots \times R_{T}$ table with ordered categories. Let $i=\left(i_{1}, \cdots, i_{T}\right)$ for $i_{k}=1, \cdots, R_{k}$ and $k=$ $1, \cdots, T$, and let $p_{i}$ denote the probability that an observation will fall in ith cell of the table. Let $X_{k}$ denote the $k$ th variable of the table for $k=1, \cdots, T$. Denote the $h$ th-order $(h=1, \cdots, T-1)$ marginal probability $P\left(X_{k_{1}}=i_{k_{1}}, \cdots, X_{k_{h}}=i_{k_{h}}\right)$ by $p_{i_{k_{1}}, \cdots, i_{k_{h}}}^{\left(k_{1}, \cdots, k_{h}\right)}$ with $1 \leq k_{1}<\cdots<k_{h} \leq T$.

In the case of $R_{1}=\cdots=R_{T} \quad(=R)$, the symmetry $\left(\mathrm{S}^{T}\right)$ model is defined by

$$
p_{i}=\psi_{i} \quad \text { for any } i \text {, }
$$

where $\psi_{i}=\psi_{j}$ for any permutation $j=\left(j_{1}, \cdots, j_{T}\right)$ of $i$ (Bhapkar and Darroch, [1]; Agresti, [2], p. 439). We may also refer to this model as the permutation-symmetry model.

The $h$ th-order marginal symmetry $\left(\mathrm{MS}_{h}^{T}\right)$ model is defined by, for a fixed $h(h=1, \cdots, T-1)$, 


$$
p_{i_{1}, \cdots, i_{h}}^{\left(s_{1}, \cdots, s_{h}\right)}=p_{j_{1}, \cdots, j_{h}}^{\left(s_{1}, \cdots, s_{h}\right)}=p_{i_{1}, \cdots, i_{h}}^{\left(t_{1}, \cdots, t_{h}\right)} \quad \text { for any }\left(i_{1}, \cdots, i_{h}\right),
$$

where $\left(j_{1}, \cdots, j_{h}\right)$ is any permutation of $\left(i_{1}, \cdots, i_{h}\right)$, and for any $\left(s_{1}, \cdots, s_{h}\right)$ and $\left(t_{1}, \cdots, t_{h}\right)$ (Bhapkar and Darroch, [1]). The $h$ th-order quasi symmetry $\left(\mathrm{QS}_{h}^{T}\right)$ model is defined by, for a fixed $h(h=1, \cdots, T-1)$,

$$
p_{i}=\mu\left(\prod_{k=1}^{T} \alpha_{k}\left(i_{k}\right)\right)\left(\prod_{1<k_{1}<k_{2} \leq T} \alpha_{k_{1} k_{2}}\left(i_{k_{1}} i_{k_{2}}\right)\right) \cdots\left(\prod_{1<k_{1}<\cdots<k_{h} \leq T} \cdots \alpha_{k_{1}, \cdots, k_{h}}\left(i_{k_{1}}, \cdots, i_{k_{h}}\right)\right) \psi_{i} \quad \text { for any } i,
$$

where $\psi_{i}=\psi_{j}$ for any permutation $j$ of $i$ (Bhapkar and Darroch, [1]). Bhapkar and Darroch [1] gave the theorem that:

1) For the $R^{T}$ table and a fixed $h(h=1, \cdots, T-1)$, the $S^{T}$ model holds if and only if both the $\mathrm{QS}_{h}^{T}$ and $\mathrm{MS}_{h}^{T}$ models hold.

Tahata, Yamamoto and Tomizawa [3] considered the $h$ th-linear ordinal quasi symmetry $\left(\operatorname{LQS}_{h}^{T}\right)$ model, which was defined by, for a fixed $h(h=1, \cdots, T-1)$,

$$
p_{i}=\mu\left(\prod_{k=1}^{T} \alpha_{k}^{i_{k}}\right)\left(\prod_{1 \leq k_{1}<k_{2} \leq T} \alpha_{k_{1} k_{2}}^{i_{k_{1}} i_{2}}\right) \cdots\left(\prod_{1 \leq k_{1}<\cdots<k_{h} \leq T} \cdots \alpha_{k_{1}, \cdots, k_{h}}^{i_{k_{1} \cdots i_{h}}}\right) \psi_{i} \quad \text { for any } i,
$$

where $\psi_{i}=\psi_{j}$ for any permutation $j$ of $i$. This model is a special case of the $\mathrm{QS}_{h}^{T}$ model. The $\mathrm{LQS}_{h}^{T}$ model is the ordinal quasi symmetry model when $h=1$ (Agresti, [4], p. 244). Tahata et al. [3] also considered the $h$ th-order marginal moment equality ( $\mathrm{MME}_{h}^{T}$ ) model, which was expressed as, for a fixed $h(h=1, \cdots, T-1)$,

$$
\mu_{k_{1}, \cdots, k_{l}}=\mu_{1, \ldots, l} \quad(l=1, \cdots, h),
$$

where $\mu_{k_{1}, \cdots, k_{l}}=E\left(X_{k_{1}} \cdots X_{k_{l}}\right)$ for $1 \leq k_{1}<\cdots<k_{l} \leq T$. Tahata et al. [3] obtained the theorem that:

2) For the $R^{T}$ table and a fixed $h(h=1, \cdots, T-1)$, the $S^{T}$ model holds if and only if both the $\operatorname{LQS}_{h}^{T}$ and $\mathrm{MME}_{h}^{T}$ models hold.

Various decompositions of the symmetry model are given by several statisticians, e.g. Caussinus [5], Bishop, Fienberg and Holland ([6], Ch.8), Read [7], Kateri and Papaioannou [8], and Tahata and Tomizawa [9].

For the $R_{1} \times R_{2} \times \cdots \times R_{T}$ table, the point-symmetry $\left(\mathrm{P}^{T}\right)$ model is defined by

$$
p_{i}=\gamma_{i} \text { for any } i \text {, }
$$

where $\gamma_{i}=\gamma_{i^{*}}$ and $i^{*}=\left(i_{1}^{*}, \cdots, i_{T}^{*}\right)$ with $i_{k}^{*}=R_{k}+1-i_{k}$ for $k=1, \cdots, T$ (Wall and Lienert, [10]; Tomizawa, [11]). This model indicates the point-symmetry of cell probabilities with respect to the center point of multi-way table.

For the $R^{T}$ table, Tahata and Tomizawa [12] considered the $h$ th-order marginal point-symmetry $\left(\mathrm{MP}_{h}^{T}\right)$ model defined by, for a fixed $h(h=1, \cdots, T-1)$,

$$
p_{i_{k_{1}}, \cdots, i_{k_{h}}}^{\left(k_{1}, \cdots k_{h}\right)}=p_{i_{k_{1}}, \cdots, i_{k_{h}}}^{\left(k_{1}, \cdots, k_{h}\right)} \quad\left(1 \leq k_{1}<\cdots<k_{h} \leq T ; i_{l}=1, \cdots, R_{l} ; l=k_{1}, \cdots, k_{h}\right) .
$$

Tahata and Tomizawa [12] also considered the $h$ th-order quasi point-symmetry ( $Q P_{h}^{T}$ ) model defined by, for a fixed $h(h=1, \cdots, T-1)$,

$$
p_{i}=\mu\left(\prod_{k=1}^{T} \alpha_{k}\left(i_{k}\right)\right)\left(\prod_{1 \leq k_{1}<k_{2} \leq T} \alpha_{k_{1} k_{2}}\left(i_{k_{1}} i_{k_{2}}\right)\right) \cdots\left(\prod_{1 \leq k_{1}<\cdots<k_{h} \leq T} \cdots \alpha_{k_{1}, \cdots, k_{h}}\left(i_{k_{1}}, \cdots, i_{k_{h}}\right)\right) \gamma_{i} \quad \text { for any } i,
$$

where $\gamma_{i}=\gamma_{i}$. Tahata and Tomizawa [12] gave the theorem that:

3) For the $R^{T}$ table and a fixed $h(h=1, \cdots, T-1)$, the $P^{T}$ model holds if and only if both the $\mathrm{QP}_{h}^{T}$ and $\mathrm{MP}_{h}^{T}$ models hold.

Theorem 3) is Theorem 1) with structures in terms of permutation-symmetry, i.e. the $\mathrm{S}^{T}, \mathrm{QS}_{h}^{T}$ and $\mathrm{MS}_{h}^{T}$ models, replaced by structures in terms of point-symmetry, i.e. the $\mathrm{P}^{T}, \mathrm{QP}_{h}^{T}$ and $\mathrm{MP}_{h}^{T}$ models. However, a theorem in terms of point-symmetry corresponding to Theorem 2) is not obtained yet. So we are now interested in the decomposition of the $\mathrm{P}^{T}$ model.

In the present paper, Section 2 proposes three models. Section 3 gives a new decomposition of the $\mathrm{P}^{T}$ model. Section 4 provides the concluding remarks. 


\section{Models}

Let $S=\left\{h \mid h=2 m-1, m=1, \cdots,\left\lfloor\frac{T}{2}\right\rfloor\right\}$, where $\lfloor x\rfloor$ denotes the largest integer less than or equal to $x$. Consider the model defined by, for a fixed odd number $h(h \in S)$,

$$
\mu_{k_{1} k_{2}, \cdots, k_{l}}=\mu_{k_{1} k_{2}, \cdots, k_{l}}^{*}\left(1 \leq k_{1}<k_{2}<\cdots<k_{l} \leq T ; l=1,3, \cdots, h\right),
$$

where

$$
\mu_{k_{1} k_{2}, \cdots, k_{l}}=E\left(X_{k_{1}} X_{k_{2}} \cdots X_{k_{l}}\right), \quad \mu_{k_{1} k_{2}, \cdots, k_{l}}^{*}=E\left(X_{k_{1}}^{*} X_{k_{2}}^{*} \cdots X_{k_{l}}^{*}\right),
$$

and $X_{k}^{*}=R_{k}+1-X_{k}$ for $k=1, \cdots, T$. We shall refer to this model as the $h$ th-order marginal moment point-symmetry ( $\mathrm{MMP}_{h}^{T}$ ) model. Note that if the $\mathrm{MP}_{h}^{T}$ model holds then the $\mathrm{MMP}_{h}^{T}$ model holds. Under the $\mathrm{MMP}_{1}^{T}$ model, we see, for any $k(k=1, \cdots, T)$,

$$
\mu_{k}=\frac{R_{k}+1}{2}
$$

Then we obtain, for any $k_{1}$ and $k_{2}\left(1 \leq k_{1}<k_{2} \leq T\right)$,

$$
\begin{aligned}
\mu_{k_{1} k_{2}}-\mu_{k_{1} k_{2}}^{*} & =\sum_{i_{k_{1}}=i_{k_{2}}=1}^{R_{k_{1}}} \sum_{k_{2}}^{R_{1}}\left(i_{k_{1}} i_{k_{2}}-i_{k_{1}}^{*} i_{k_{2}}^{*}\right) p_{i_{k_{1}} i_{k_{2}}}^{\left(k_{1}, k_{2}\right)} \\
& =-\left(R_{k_{1}}+1\right)\left(R_{k_{2}}+1\right)+\left(R_{k_{1}}+1\right) \mu_{k_{2}}+\left(R_{k_{2}}+1\right) \mu_{k_{1}}=0 .
\end{aligned}
$$

Under the $\mathrm{MMP}_{3}^{T}$ model, we see, for any $k_{1}, k_{2}$ and $k_{3}\left(1 \leq k_{1}<k_{2}<k_{3} \leq T\right)$,

$$
\mu_{k_{1} k_{2} k_{3}}=-\frac{1}{2}\left(\frac{1}{2}\left(R_{k_{1}}+1\right)\left(R_{k_{2}}+1\right)\left(R_{k_{3}}+1\right)-\left(R_{k_{1}}+1\right) \mu_{k_{2} k_{3}}-\left(R_{k_{2}}+1\right) \mu_{k_{1} k_{3}}-\left(R_{k_{3}}+1\right) \mu_{k_{1} k_{2}}\right) \text {. }
$$

Then we obtain, for any $k_{1}, k_{2}, k_{3}$ and $k_{4}\left(1 \leq k_{1}<k_{2}<k_{3}<k_{4} \leq T\right)$,

$$
\begin{aligned}
& \left.\mu_{k_{1} k_{2} k_{3} k_{4}}-\mu_{k_{1} k_{2} k_{3} k_{4}}^{*}=\sum_{i_{k_{1}}=\sum_{k_{2}}=1 i_{k_{3}}=\sum_{k_{4}}=1}^{R_{k_{1}}} \sum_{k_{k_{2}}}^{R_{k_{3}}} \sum_{k_{k_{1}}}^{R_{k_{1}}} i_{k_{2}} i_{k_{3}} i_{k_{4}}-i_{k_{1}}^{*} i_{k_{2}}^{*} i_{k_{3}}^{*} i_{k_{4}}^{*}\right) p_{i_{k_{1}} k_{2} k_{2} i_{3} k_{k_{4}}}^{\left(k_{1}, k_{4}, k_{4}, k_{4}\right)} \\
& =\left(R_{k_{1}}+1\right)\left(R_{k_{2}}+1\right)\left(R_{k_{3}}+1\right)\left(R_{k_{4}}+1\right)-\left(R_{k_{1}}+1\right)\left(R_{k_{2}}+1\right) \mu_{k_{3} k_{4}} \\
& -\left(R_{k_{1}}+1\right)\left(R_{k_{3}}+1\right) \mu_{k_{2} k_{4}}-\left(R_{k_{1}}+1\right)\left(R_{k_{4}}+1\right) \mu_{k_{2} k_{3}}-\left(R_{k_{2}}+1\right)\left(R_{k_{3}}+1\right) \mu_{k_{1} k_{4}} \\
& -\left(R_{k_{2}}+1\right)\left(R_{k_{4}}+1\right) \mu_{k_{1} k_{3}}-\left(R_{k_{3}}+1\right)\left(R_{k_{4}}+1\right) \mu_{k_{1} k_{2}}+\left(R_{k_{1}}+1\right) \mu_{k_{2} k_{3} k_{4}} \\
& +\left(R_{k_{2}}+1\right) \mu_{k_{1} k_{3} k_{4}}+\left(R_{k_{3}}+1\right) \mu_{k_{1} k_{2} k_{4}}+\left(R_{k_{4}}+1\right) \mu_{k_{1} k_{2} k_{3}} \\
& =0 \text {. }
\end{aligned}
$$

Thus we are not interested in the $\mathrm{MMP}_{h}^{T}$ model with $h$ being even. Therefore we shall consider the $\mathrm{MMP}_{h}^{T}$ model with $h$ being odd.

Consider the model defined by

$$
p_{i}=\mu\left(\prod_{k=1}^{T} \alpha_{k}^{i_{k}}\right) \gamma_{i} \text { for any } i,
$$

where $\gamma_{i}=\gamma_{i}{ }^{*}$. We shall refer to this model as the ordinal quasi point-symmetry $\left(\mathrm{OQP}^{T}\right)$ model. In the case of $T=2$, this model is identical to the model proposed by Tahata and Tomizawa [13]. The special case of the $\mathrm{OQP}^{T}$ model obtained by putting $\alpha_{1}=\cdots=\alpha_{T}=1$ is the $\mathrm{P}^{T}$ model. Also the $\mathrm{OQP}^{T}$ model is the special case of the $\mathrm{QP}_{1}^{T}$ model obtained by putting $\left\{\alpha_{k}\left(i_{k}\right)=\alpha_{k}^{i_{k}}\right\}$. The $\mathrm{OQP}^{T}$ model may be expressed as

$$
\log \frac{p_{i}}{p_{i^{*}}}=\beta_{0}+\sum_{k=1}^{T} i_{k} \beta_{k} \quad \text { for any } i \text {, }
$$

with $\beta_{0}=-\sum_{k}\left(R_{k}+1\right) \log \alpha_{k}$ and $\beta_{k}=\log \alpha_{k}^{2}$. From this equation, we can see the log-odds that an ob- 
servation falls in ith cell instead of in the point-symmetric $i^{*}$ th cell, i.e. $\log \left(p_{i} / p_{i^{*}}\right)$, is described as a linear combination with intercept $\beta_{0}$ and slope $\beta_{k}$ for the category indicator $i_{k}$ under the OQP ${ }^{T}$ model. Thus the parameter $\beta_{k}$ can be interpreted as the effect of a unit increase in the $k$ th variable on the log-odds.

Consider the model being more general than the $\mathrm{OQP}^{T}$ model as follows, for a fixed odd number $h(h \in S)$,

$$
p_{i}=\mu\left(\prod_{k=1}^{T} \alpha_{k}^{i_{k}}\right)\left(\prod_{1 \leq k_{1}<k_{2}<k_{3} \leq T} \alpha_{k_{1} k_{2} k_{3}}^{i_{k_{1}} i_{k} i_{k_{3}}}\right) \cdots\left(\prod_{1 \leq k_{1}<\cdots<k_{h} \leq T} \cdots \alpha_{k_{1}, \cdots, k_{h}}^{i_{k_{1}}, \cdots k_{k_{n}}}\right) \gamma_{i} \quad \text { for any } i,
$$

where $\gamma_{i}=\gamma_{i^{*}}$. We shall refer to this model as the $h$ th-linear ordinal quasi point-symmetry $\left(\mathrm{LQP}_{h}^{T}\right)$ model. Especially, when $h=1$, the $\mathrm{LQP}_{h}^{T}$ model is identical to the $\mathrm{OQP}^{T}$ model. Also the $\mathrm{LQP}_{h}^{T}$ model is the special case of the $\mathrm{QP}_{h}^{T}$ model obtained by putting

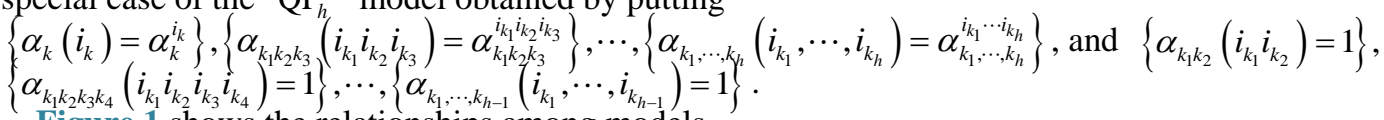

Figure 1 shows the relationships among models.

\section{Decomposition of Point-Symmetry}

We obtain the following theorem:

Theorem 1. For the $R_{1} \times R_{2} \times \cdots \times R_{T}$ table and a fixed odd number $h(h \in S)$, the $P^{T}$ model holds if and only if both the $L Q P_{h}^{T}$ and $M M P_{h}^{T}$ models hold.

Proof. If the $\mathrm{P}^{T}$ model holds, then both the $L Q P_{h}^{T}$ and $M M P_{h}^{T}$ models hold. Assuming that both the $L Q P_{h}^{T}$ and $M M P_{h}^{T}$ models hold, then we shall show the $\mathrm{P}^{T}$ model holds. Let $q=\left\{q_{i}\right\}$ denote cell probabilities which satisfy both the $L Q P_{h}^{T}$ and $M M P_{h}^{T}$ models. The $L Q P_{h}^{T}$ model is expressed as

$$
\log q_{i}=\log \mu \gamma_{i}+\sum_{k=1}^{T} i_{k} \log \alpha_{k}+\sum_{1 \leq k_{1}<k_{2}<k_{3} \leq T} \sum_{k_{1}} i_{k_{2}} i_{k_{3}} \log \alpha_{k_{1} k_{2} k_{3}}+\cdots+\sum_{1 \leq k_{1}<\cdots<k_{h} \leq T} \cdots i_{k_{1}} \cdots i_{k_{h}} \log \alpha_{k_{1}, \cdots, k_{h}},
$$

where $\gamma_{i}=\gamma_{i}$. Let

$$
C=\sum_{i_{1}=1}^{R_{1}} \cdots \sum_{i_{T}=1}^{R_{T}} \gamma_{i}, \quad \pi_{i}=\frac{\gamma_{i}}{C} .
$$

Note that $\pi=\left\{\pi_{i}\right\}$ satisfy $0<\pi_{i}<1, \quad \sum_{i_{1}} \cdots \sum_{i_{T}} \pi_{i}=1$ and $\pi_{i}=\pi_{i^{*}}$. Then the $\operatorname{LQP}_{h}^{T}$ model is also expressed as

$$
\begin{aligned}
\log \left(\frac{q_{i}}{\pi_{i}}\right)= & \log \mu c+\sum_{k=1}^{T} i_{k} \log \alpha_{k}+\sum_{1 \leq k_{1}<k_{2}<k_{3} \leq T} i_{k_{1}} i_{k_{2}} i_{k_{3}} \log \alpha_{k_{1} k_{2} k_{3}} \\
& +\cdots+\sum_{1 \leq k_{1}<\cdots<k_{h} \leq T} \cdots i_{k_{1}} \cdots i_{k_{h}} \log \alpha_{k_{1}, \cdots, k_{h}} .
\end{aligned}
$$

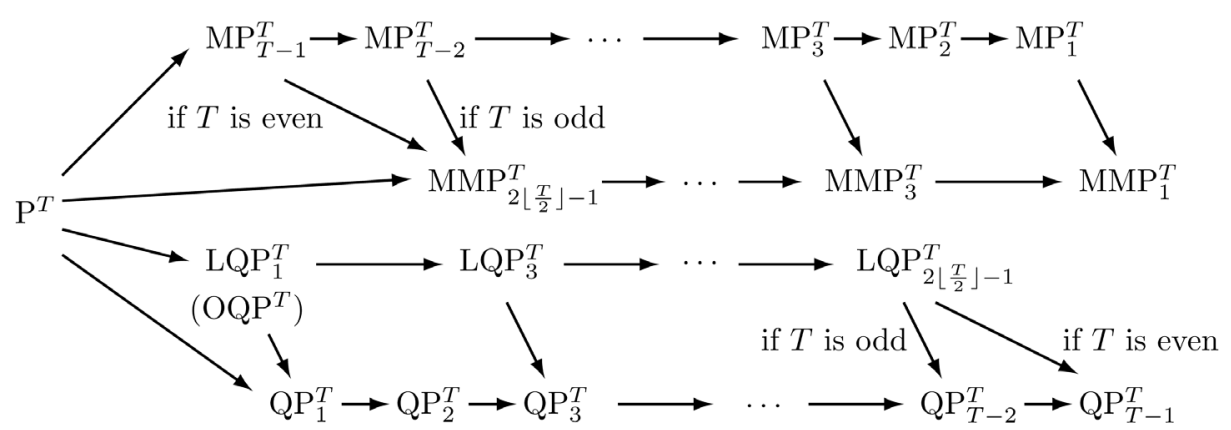

Figure 1. Relationships among various models. Note: “ $M_{1} \rightarrow M_{2}$ ” indicates that model $M_{1}$ implies model $M_{2}$. 
The $\mathrm{MMP}_{h}^{T}$ model is expressed as

$$
\mu_{k_{1} k_{2}, \cdots, k_{l}}^{q}=\mu_{k_{1} k_{2}, \cdots, k_{l}}^{q^{*}} \quad\left(1 \leq k_{1}<k_{2}<\cdots<k_{l} \leq T ; l=1,3, \cdots, h\right),
$$

where

$$
\mu_{k_{1}, k_{2}, \cdots, k_{l}}^{q}=\sum_{i_{k_{1}}=1}^{R_{k_{1}}} \cdots \sum_{i_{k_{l}}=1}^{R_{k_{l}}} i_{k_{1}} \cdots i_{k_{l}} q_{i_{k_{1}}, \cdots, i_{k_{l}}}^{\left(k_{1}, \cdots, k_{l}\right)}, \quad \mu_{k_{1} k_{2}, \ldots, k_{l}}^{q^{*}}=\sum_{i_{k_{1}}=1}^{R_{k_{1}}} \cdots \sum_{i_{k_{l}}=1}^{R_{k_{l}}} i_{k_{1}}^{*} \cdots i_{k_{l}}^{*} q_{i_{k_{1}}, \cdots, i_{k_{l}}}^{\left(k_{1}, \cdots, k_{l}\right)} .
$$

Then we denote $\mu_{k_{1} k_{2}, \cdots, k_{l}}^{q}\left(=\mu_{k_{1} k_{2}, \cdots, k_{l}}^{q^{*}}\right)$ by $\mu_{k_{1} k_{2}, \cdots, k_{l}}^{0}$.

Consider arbitrary cell probabilities $p=\left\{p_{i}\right\}$ which satisfy the $\mathrm{MMP}_{h}^{T}$ model and

$$
\mu_{k_{1} k_{2}, \cdots, k_{l}}^{p}=\mu_{k_{1} k_{2}, \cdots, k_{l}}^{p^{*}}=\mu_{k_{1} k_{2}, \cdots, k_{l}}^{0} \quad\left(1 \leq k_{1}<k_{2}<\cdots<k_{l} \leq T ; l=1,3, \cdots, h\right),
$$

where

$$
\mu_{k_{1}, k_{2}, \cdots, k_{l}}^{p}=\sum_{i_{k_{1}}=1}^{R_{k_{1}}} \cdots \sum_{i_{k_{l}}=1}^{R_{k_{l}}} i_{k_{1}} \cdots i_{k_{l}} p_{i_{k_{1}}, \cdots, i_{k_{l}}}^{\left(k_{1}, \cdots, k_{l}\right)}, \quad \mu_{k_{1} k_{2}, \cdots, k_{l}}^{p *}=\sum_{i_{k_{1}}=1}^{p_{k_{1}}} \cdots \sum_{i_{k_{l}}=1}^{R_{k_{l}}} i_{k_{1}}^{*} \cdots i_{k_{l}}^{*} p_{i_{k_{1}}, \cdots, k_{k_{l}}}^{\left(k_{1}, \cdots, k_{l}\right)}
$$

From (1), (2) and (3),

$$
\sum_{i_{1}=1}^{R_{1}} \cdots \sum_{i_{T}=1}^{R_{T}}\left(p_{i}-q_{i}\right) \log \left(\frac{q_{i}}{\pi_{i}}\right)=0 .
$$

Let $K(\because \because)$ denote the Kullback-Leibler information, e.g., it between $q$ and $\pi$ is

$$
K(q ; \pi)=\sum_{i_{1}=1}^{R_{1}} \cdots \sum_{i_{T}=1}^{R_{T}} q_{i} \log \left(\frac{q_{i}}{\pi_{i}}\right) .
$$

From (4),

$$
K(p ; \pi)=K(p ; q)+K(q ; \pi) .
$$

Thus, for fixed $\pi$,

$$
K(q ; \pi)=\min _{p} K(p ; \pi),
$$

and then $q$ uniquely minimize $K(p ; \pi)$ (see Darroch and Ratcliff, [14]).

Let $q^{*}=\left\{q_{i^{*}}\right\}$. Then, in a similar way as described above, we obtain

$$
K\left(q^{*} ; \pi\right)=\min _{p} K(p ; \pi),
$$

and then $q^{*}$ uniquely minimize $K(p ; \pi)$, hence $q=q^{*}$. Namely $q$ satisfy the $\mathrm{P}^{T}$ model. The proof is completed.

For the analysis of data, the test of goodness-of-fit of the $\mathrm{LQP}_{h}^{T}$ model is achieved based on, e.g., the likelihood ratio chi-square statistic which has a chi-square distribution with the number of degrees of freedom

$$
\begin{cases}\frac{1}{2}\left(\prod_{k=1}^{T} R_{k}-1\right)-\sum_{i=1}^{\frac{h+1}{2}}\left(\begin{array}{c}
T \\
2 i-1
\end{array}\right) & \left(R_{k}: \text { odd for } k=1, \cdots, T\right), \\
\frac{1}{2} \prod_{k=1}^{T} R_{k}-\sum_{i=1}^{\frac{h+1}{2}}\left(\begin{array}{c}
T \\
2 i-1
\end{array}\right) & \text { (otherwise). }\end{cases}
$$

Also the number of degrees of freedom for the $\mathrm{MMP}_{h}^{T}$ model is

$$
\sum_{i=1}^{\frac{h+1}{2}}\left(\begin{array}{c}
T \\
2 i-1
\end{array}\right) \text {. }
$$


We point out that, for a fixed $h$, the number of degrees of freedom for the $\mathrm{P}^{T}$ model is equal to sum of those for the $\mathrm{LQP}_{h}^{T}$ and $\mathrm{MMP}_{h}^{T}$ models.

\section{Concluding Remarks}

For multi-way contingency tables, we have proposed the $\mathrm{MMP}_{h}^{T}, \mathrm{OQP}^{T}$ and $\mathrm{LQP}_{h}^{T}$ models. Under the $\mathrm{OQP}^{T}$ model, the log-odds that an observation falls in a cell instead of in its point-symmetric cell is described as a linear combination of category indicators. For a fixed odd number $h(h \in S)$, the $\mathrm{LQP}_{h}^{T}$ model implies the $\mathrm{QP}_{h}^{T}$ model.

We have gave the theorem that the $\mathrm{P}^{T}$ model holds if and only if both the $\mathrm{LQP}_{h}^{T}$ and $\mathrm{MMP}_{h}^{T}$ models. For the analysis of data, the decomposition given in the present paper may be useful for determining the reason when the $\mathrm{P}^{T}$ model fits data poorly.

\section{Acknowledgements}

The authors thank the editor and the referees for their helpful comments.

\section{References}

[1] Bhapkar, V.P. and Darroch, J.N. (1990) Marginal Symmetry and Quasi Symmetry of General Order. Journal of Multivariate Analysis, 34, 173-184. http://dx.doi.org/10.1016/0047-259X(90)90034-F

[2] Agresti, A. (2013) Categorical Data Analysis. 3rd Edition, Wiley, Hoboken.

[3] Tahata, K., Yamamoto, H. and Tomizawa, S. (2011) Linear Ordinal Quasi-Symmetry Model and Decomposition of Symmetry for Multi-Way Tables. Mathematical Methods of Statistics, 20, 158-164. http://dx.doi.org/10.3103/S1066530711020050

[4] Agresti, A. (2010) Analysis of Ordinal Categorical Data. 2nd Edition, Wiley, Hoboken. http://dx.doi.org/10.1002/9780470594001

[5] Caussinus, H. (1965) Contribution à l'analyse statistique des tableaux de corrélation. Annales de la Faculté des Sciences de l'Université de Toulouse, 29, 77-182. http://dx.doi.org/10.5802/afst.519

[6] Bishop, Y.M.M., Fienberg, S.E. and Holland, P.W. (1975) Discrete Multivariate Analysis: Theory and Practice. MIT Press, Cambridge.

[7] Read, C.B. (1977) Partitioning Chi-Square in Contingency Tables: A Teaching Approach. Communications in Statistics, Theory and Methods, 6, 553-562. http://dx.doi.org/10.1080/03610927708827513

[8] Kateri, M. and Papaioannou, T. (1997) Asymmetry Models for Contingency Tables. Journal of the American Statistical Association, 92, 1124-1131. http://dx.doi.org/10.1080/01621459.1997.10474068

[9] Tahata, K. and Tomizawa, S. (2014) Symmetry and Asymmetry Models and Decompositions of Models for Contingency Tables. SUT Journal of Mathematics, 50, 131-165.

[10] Wall, K. and Lienert, G.A. (1976) A Test for Point-Symmetry in J-Dimensional Contingency-Cubes. Biometrical Journal, 18, 259-264.

[11] Tomizawa, S. (1985) The Decompositions for Point Symmetry Models in Two-Way Contingency Tables. Biometrical Journal, 27, 895-905. http://dx.doi.org/10.1002/bimj.4710270811

[12] Tahata, K. and Tomizawa, S. (2008) Orthogonal Decomposition of Point-Symmetry for Multiway Tables. Advances in Statistical Analysis, 92, 255-269. http://dx.doi.org/10.1007/s10182-008-0070-5

[13] Tahata, K. and Tomizawa, S. (2015) Ordinal Quasi Point-Symmetry and Decomposition of Point-Symmetry for CrossClassifications. Journal of Statistics: Advances in Theory and Applications, 14, 181-194.

[14] Darroch, J.N. and Ratcliff, D. (1972) Generalized Iterative Scaling for Log-Linear Models. Annals of Mathematical Statistics, 43, 1470-1480. http://dx.doi.org/10.1214/aoms/1177692379 